\title{
Characteristics of the F response: a single motor unit study
}

\author{
STEPHEN K. YATES AND WILLIAM F. BROW N \\ From the University Hospital, London, Ontario, Canada
}

SUMMARY Trains of 100 to 200 stimuli result in F discharges from less than one half of motor units of hand muscles. The maximum observed $\mathrm{F}$ discharge frequency was $10 \%$. There was no relation between the surface voltage of motor unit potentials and the frequency of $F$ discharge. The motor unit potentials of larger surface voltage were recruited at higher stimulus intensity levels, usually supramaximal for the antidromic sensory nerve action potential. No correlation was observed between $\mathrm{F}$ latency and the surface voltage of the motor unit potentials. In only one pathological example was a clear interaction observed between the frequency of $F$ discharge in motor unit potentials and the recruitment of other single motor unit potentials by increments in the stimulus intensity. The observations suggested that caution should be exercised before the $\mathrm{F}$ discharge is accepted as a method for measuring proximal conduction times in human motor nerves.

The $\mathrm{F}$ motoneurone discharge has been used to measure conduction times over proximal segments of nerves (Kimura, 1974; Kimura et al., 1974; Kimura and Butzer, 1975; King and Ashby, 1976; Eisen et al., 1977a, b). The method must, however, take account of known characteristics of the $\mathrm{F}$ discharge. The $\mathrm{F}$ discharge, for example, represents the summation of the recurrent discharges of individual motor unit potentials (MUPs) that vary in voltage and latency from MUP to MUP, even though the latencies for any particular MUP remain remarkably constant from stimulus to stimulus (Bergmans, 1970; Trontelj, 1973a, b; Trontelj and Trontelj, 1973; Feasby and Brown, 1974; Schiller and Stalberg, 1977, 1978). Moreover, the $F$ discharge frequency for individual MUPs is low (Bergmans, 1970; Feasby and Brown, 1974; Schiller and Stalberg, 1978).

These observations made it critically important to have a better understanding of the factors that determine the chance of F discharge in MUPs. For example, it is particularly important to know if the motor units with shortest latency $F$ responses are truly representative of the nerve fibres with maximum motor conduction velocities across proximal nerve segments. In addition, it is im-

Address for reprint requests: Dr W. F. Brown, Department of Clinical Neurological Sciences, University Hospital, London, Ontario, Canada N6A 5A5.

Accepted 10 August 1978 portant to know if increments or decrements in the stimulus intensity change the likelihood of $F$ discharge in MUPs, what the relation of frequency of $F$ discharge is to the MUP surface voltage and the relation of MUP surface voltage to the $F$ wave latency.

Methods previously used (Feasby and Brown, 1974), together with more recent techniques for investigating single MUPs at threshold (Kadrie et al., 1976; Kadrie and Brown, 1978), were chosen to look at the above questions. The results suggested that caution should be exercised in the acceptance of the $F$ discharge as a method for measuring proximal conduction times in human nerves.

\section{Subjects and methods}

The results described were obtained from healthy volunteers between $25-40$ years of age, except for one example from the ulnar nerve of a patient with Guillain-Barré polyneuritis.

To investigate $\mathrm{F}$ discharges with stimulation at, or close to, motor threshold, the multiple point stimulation method was used (Kadrie et al., 1976; Kadrie and Brown, 1978). The original intention to relate MUP peak-to-peak voltage (p-pV) to the respective $M$ and $F$ latencies failed because only a statistically insignificant number (four) of stimulation points were encountered where the 
thresholds of the first two to four MUPs were sufficiently separate and $F$ discharges occurred in at least two or more of the motor units to permit the intended comparisons.

The effect of changes in the stimulus intensity on the $F$ discharge was tested on 10 ulnar nerves and two median nerves. The nerves were stimulated at the level of the wrist at a rate of one per second, and at intensities sufficient to evoke direct $M$ responses from the respective hypothenar or thenar muscles 10, 25, 50, and $100 \%$ of the maximum compound muscle potentials. At each level of stimulus intensity 100 or 200 stimuli were delivered. The surface voltages of single MUPs in the $F$ discharge at the respective fixed stimulus intensity levels were compared. Except at or just above motor threshold and even with the use of the single fibre EMG electrode it was not possible, in our experience, to compare directly unit discharges from the direct $M$ and late $F$ responses. Therefore, to be accepted as a true single MUP, MUPs from the $F$ discharge were required to meet three criteria: (1) the potential repeated and was identical in configuration and surface voltage, (2) the potential varied in latency by less than 100 microseconds, and (3) the likelihood was negligible that the potential represented the sum of two or more MUPs.

The last criterion was based on the assumption first put forward by one of us in 1974 (Feasby and Brown, 1974) that the chance of $F$ discharge for any motor unit was independent of the $F$ discharge of other motor units. If true, the probable discharge frequency for the sum of two or more MUPs would equal the product of the discharge frequencies of the component MUPs. Therefore, provided unit potentials meeting the first two criteria did not have "subunits" of appropriate latency, surface voltage or F discharge frequency to account for the units in question, the MUP was accepted as representing the voltage from a single MUP.

By comparison of the surface voltages of MUPs in the $F$ discharges at various levels of stimulus intensity, it was our hope to learn firstly if the frequency of $F$ recurrent discharge in MUPs could be changed by changes in the stimulus intensity level, and secondly if MUPs were recruited in any particular order of surface voltage or latency by changes in the stimulus intensity.

To compare the relative theshold of sensory and motor fibres, the evoked muscle (hypothenar or thenar) compound potential and corresponding antidromic fifth or first antidromic digital sensory nerve action potentials were measured over a range of stimulus intensities. Changes in the $F$ discharge of MUPs at stimulus intensities which were supramaximal for the antidromic digital sensory nerve action potential were judged not to have resulted from conditioning inputs from sensory fibres. To look further at this last proposition, a preconditioning sensory input evoked by stimulation of an appropriate digit was timed to precede the stimulus delivered to the motor nerve by intervals from 0-16 ms. The time between the conditioning digital inputs and the test motor stimulus was reduced by intervals of $2 \mathrm{~ms}$, at each interval 100 test stimuli being delivered to the motor nerve to evoke a constant $\mathrm{M}$ discharge in the test motor unit.

In one patient with Guillain-Barré polyneuritis (absent sensory nerve potentials in the upper limb) it was possible to examine changes in the frequency of the $F$ discharge of one MUP over a range of stimulus intensities at which two other MUPs were excited. This pathological situation provided us with our only opportunity in healthy or abnormal nerves, to test satisfactorily and reproducibly for changes in frequency of $F$ discharge in a single MUP in response to the recruitment of other single MUPs.

\section{Results}

PROPORTION OF MOTOR UNIT POTENTIALS WITH AN F RESPONSE

For trains of 100 stimuli, the percentage of motor units that had an $\mathrm{F}$ response varied from nil $(0: 9)$ in one subject, to $45 \%(5: 11)$ in another subject, the mean being $23 \%$ for all control nerves and motor units. This percentage was nearly identical

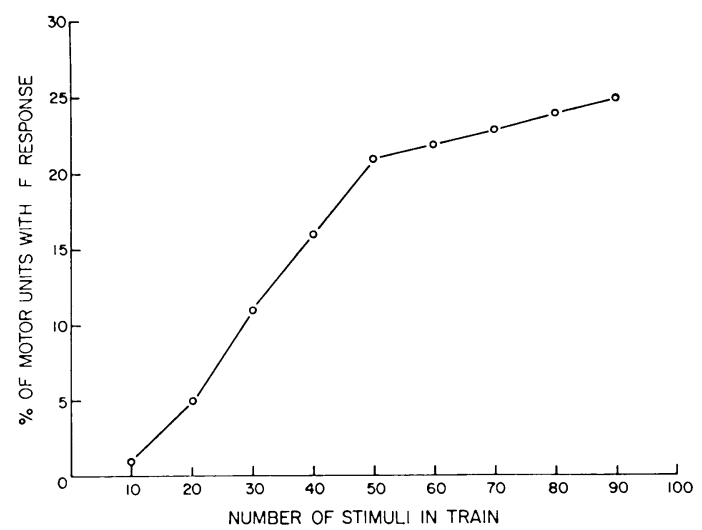

Fig. 1 Percentage of motor unit potentials (MUPs) with an $F$ discharge in relation to the number of stimuli in the train (10-100), all stimuli evoking a direct $M$ response in the respective single motor units. Stimulus frequency was one per second. 
for MUPs recruited near the level of the wrist $(22.5 \%, 40$ MUPs in total) as for MUPs recruited at the elbow level $(20 \%, 70$ MUPs in total). The more stimuli in the train, the larger was the fraction of motor units with an $F$ response. For example, less than $5 \%$ of motor units excited at or close to motor threshold had $\mathrm{F}$ discharges if the stimulus train was less than 20. An increase in the number of stimuli to 50 , increased the proportion of units with an $\mathrm{F}$ discharge to over $20 \%$. Further increases in the length of stimulus trains did not significantly increase the fraction of motor units with an $F$ discharge (Fig. 1). These observations suggested that a train of at least 50 stimuli is necessary to observe $F$ discharges in most of the motor units capable of generating a recurrent action potential. At or close to motor threshold the maximum observed $\mathrm{F}$ discharge frequency was $10 \%$ in control motor units.

RELATION BETWEEN THE CHANCE OF MOTOR UNIT F DISCHARGE AND THE MOTOR UNIT POTENTIAL SURFACE VOLTAGE

A total of 110 thenar MUPs could be isolated by carefully grading the stimulus intensity delivered to the nerves (nine control median nerves). Of this total, 70 were the first MUPs excited above threshold; the problem of overlapping motor unit firing levels limited to 30 the number of second MUPs and 10 third MUPs that could be isolated above threshold (Brown and Milner-Brown, 1976). If second and third MUPs are included, the range and distribution of the surface $\mathrm{p}-\mathrm{pV}$ for MUPs obtained in this manner closely approximates that for isometric contraction, successively recruited MUPs having larger surface voltages (Fig. 2). The chance of $F$ discharge was not related to the order of recruitment of the motor units or the surface voltage of the MUPs; nearly equal percentages of small, $(<0.05 \mathrm{mV})$, medium $(0.05-$ $0.149 \mathrm{mV})$, and large $(>0.150 \mathrm{mV})$ MUPs having $F$ discharges.

\section{EFFECT OF THE INTENSITY OF NERVE STIMULATION} ON THE F DISCHARGE

Motor units recruited into the $\mathrm{F}$ discharge by constant intensity stimuli sufficient to evoke direct $\mathbf{M}$ responses, 1025,50 , and $100 \%$ of the maximum compound potential had potentials with significantly larger surface voltages at successive increment levels in the stimulus intensity. At times, MUPs recruited at lower stimulus intensity levels were absent from the $\mathrm{F}$ discharge at higher stimulus intensity levels. The latter observation was

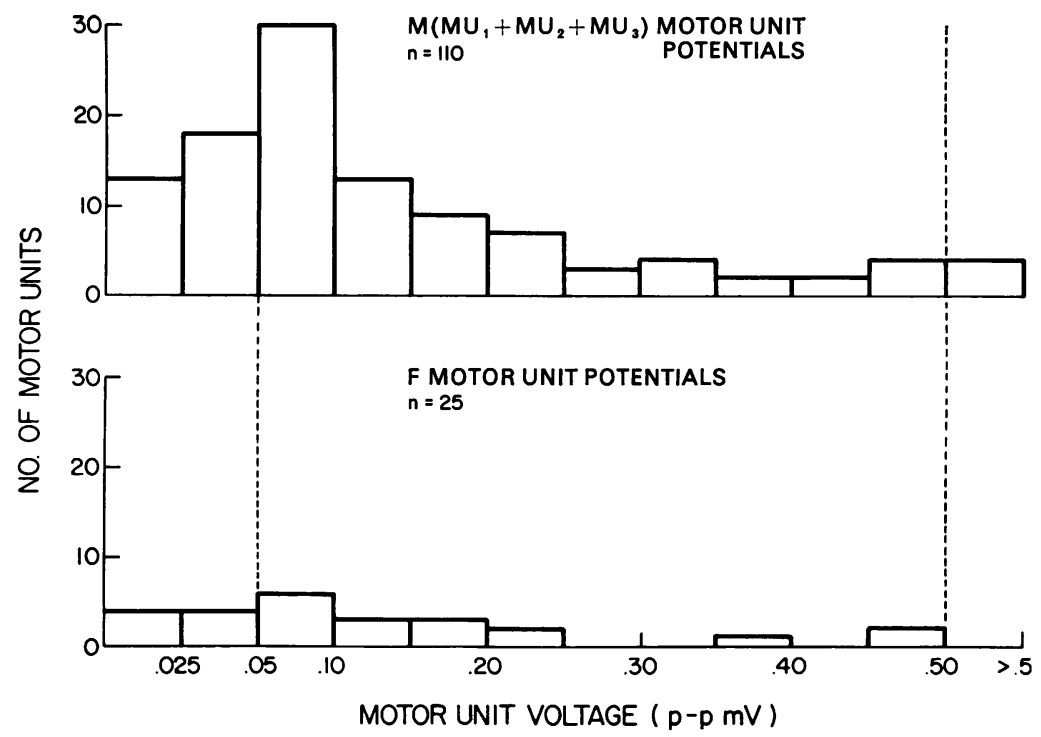

Fig. 2 Upper: voltage distribution for MUPs recruited first (MU1), second (MU2), or third (MU3) at or just above threshold by increments in the stimulus intensity. Surface p-pV $(m V)-M U 10.136 \pm 0.1361 S D ; M U 2$ $0.133 \pm 0.1931 S D ; M U 30.253 \pm 0.1361 S D$. Lower: surface voltage distribution of MUPs from above population of MUPs with an F discharge. Note changes in the scale factors at 0.05 and $0.5 \mathrm{mV}$ : Stimulus was delivered at one per second. 
particularly evident if the surface voltage distribution for the first MUPs excited above motor threshold (Fig. 2, upper) was compared with the corresponding surface voltage distributions for F MUPs recruited at higher stimulus intensity levels (Fig 3). Depending on the motor unit, increases or reductions in $F$ motor unit discharge frequency were observed in response to changes in the stimulus intensity level as illustrated by Fig. 4 .

FACTORS THAT CHANGE THE FREQUENCY OF THE F MOTOR UNIT POTENTIAL DISCHARGE

In healthy nerves, changes in the discharge frequency of F MUPs frequently occurred at stimulus intensities clearly above levels evoking maximum antidromic digital sensory nerve action potentials. For example, in Fig. 5, the $M$ wave was only $15 \%$ of the muscle maximum compound potential at a stimulus intensity that evoked a maximum antidromic sensory nerve action potential. It is reasonable to think that at this stimulus intensity all the sensory fibres with a chance of reaching the spinal cord at or just before the antidromic motor volley, have been excited. Higher threshold

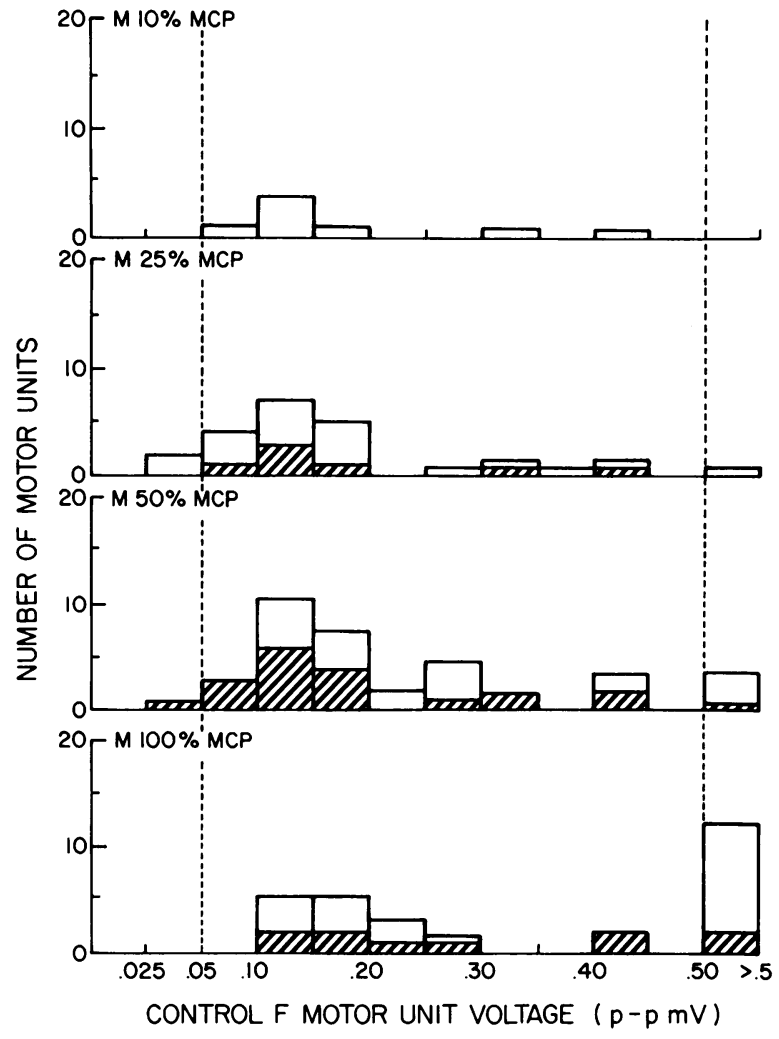

sensory fibres probably had too long a latency to condition the motoneurones at or just before the arrival of the antidromic motor impulses. This view, that inputs from large sensory fibres fail to change the chance for $F$ discharge, was supported by the observation that supramaximal digital nerve stimulation failed to change the frequency of $F$ discharge in MUPs even when the sensory fibre input was timed to arrive at the spinal cord up to $16 \mathrm{~ms}$ earlier than the antidromic motor input.

The above observations, though not conclusively proving that sensory input failed to change the frequency of $F$ motor neurone discharge, clearly suggested that such an effect, if present, was marginal. On the contrary, our observations suggested that it was more likely that changes in the frequency of $\mathrm{F}$ motor unit discharge could be brought about by antidromic inputs from other motor fibres, to account for the observations illustrated by Figs. 3 and 4 . For such an action to occur the antidromic input from certain motor fibres would have to arrive at the spinal cord before the arrival of antidromic inputs from lower conduction velocity motor axons. It has not been

Fig. 3 Distribution of the surface voltages of $F$ motor unit potentials in millivolts $(\mathrm{mV})$ at stimulus intensities evoking $M$ direct responses 10, 25, 50, and $100 \%$ of the maximum compound potential (MCP). Significant differences between F MUPs at the different levels of stimulus intensity were observed $(P<0.05)$. Cross-hatched bars represent those MUPs present not only at that stimulus intensity level but at next lower level of stimulus intensity. Note the changes in the voltage scale factor at 0.05 and $0.50 \mathrm{mV}$. Stimulus frequency was one per second. 


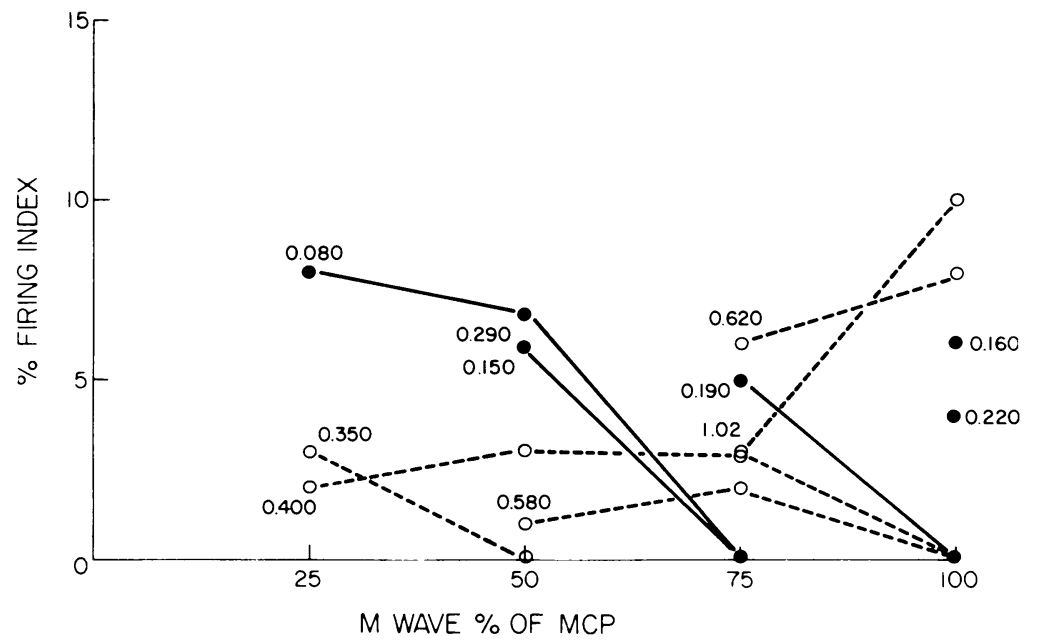

Fig. 4 A representative control ulnar nerve. Illustrated are the surface voltages (see figures in millivolts) of MUPs recruited into the F discharge by trains of 200 stimuli at intensity levels sufficient to evoke a direct $M$ response $25,50,75$, and $100 \%$ of the hypothenar MCP. The F discharge frequency $(\%$ index) changed up or down in response to changes in the stimulus intensity in different MUPs. Overall, in all subjects, MUPs recruited at higher stimulus intensity levels had larger surface voltages, the discharge frequency increasing with increments in the stimulus intensity. MUPS recruited at lower stimulus intensity levels were usually of lower surface voltage, reductions in the $F$ discharge frequency being observed in response to increments in the stimulus intensity. Stimulus frequency was one per second.

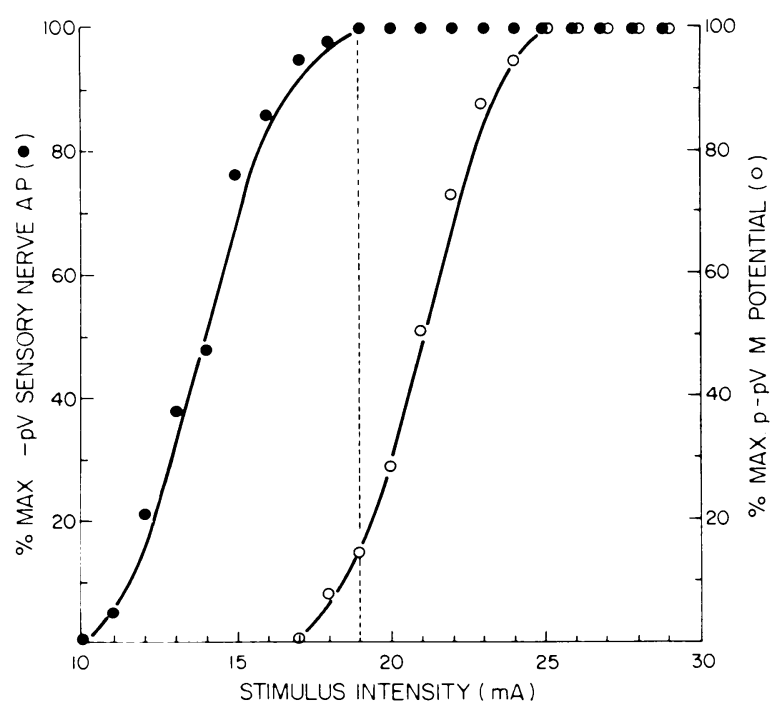

Fig. 5 The fifth digit antidromic sensory nerve action potential negative peak $(-p)$ voltage in relation to the hypothenar surface voltage evoked by changes in the intensity of stimuli delivered to the ulnar nerve at the level of the wrist. Note that the antidromic sensory nerve action potential voltage was maximum when the $M$ discharge was less than $20 \%$ of the maximum compound potential voltage. Stimulus duration was $0.1 \mathrm{~ms}$.

possible to provide direct support for this theoretical prediction up to now. In support, however, was the observation that as larger MUPs were recruited into the $F$ discharge at higher stimulus intensity levels, reductions in the $\mathrm{F}$ discharge fre- quency in some of the lower surface voltage MUPs were observed. The latter, at least in the direct $\mathbf{M}$ response, have longer latencies and could, therefore, be conditioned by antidromic inputs from shorter latency motor fibres. 


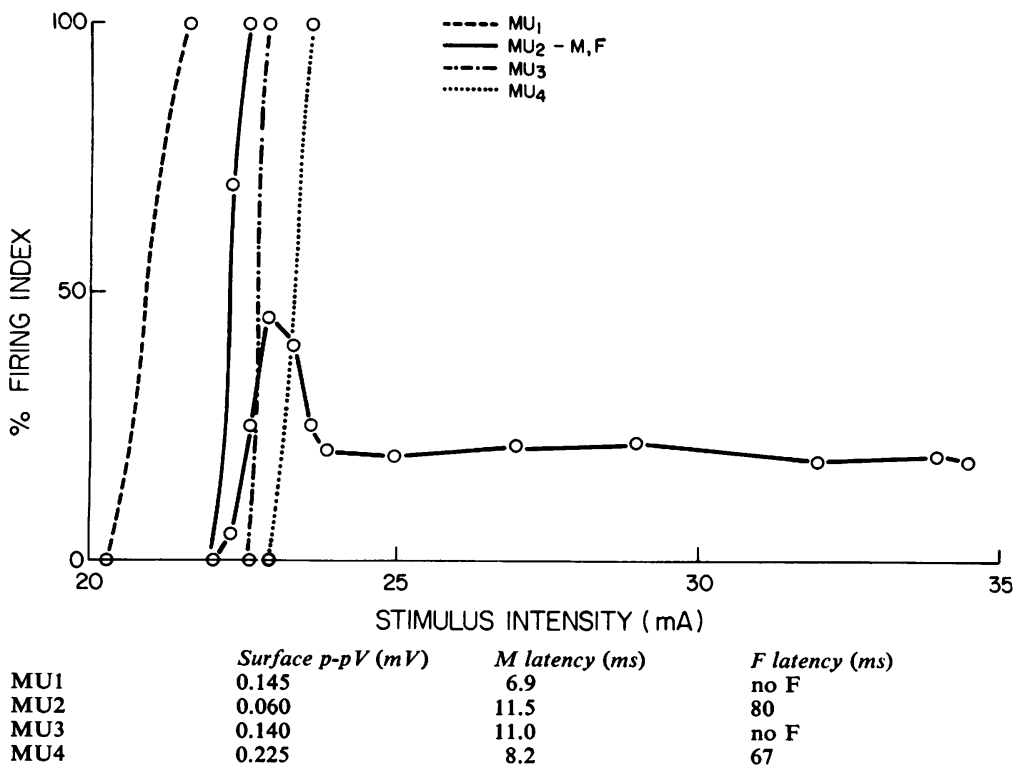

Fig. 6 Changes in the $F$ discharge frequency of the second MUP (MU2) recruited above motor threshold by further increments in the stimulus intensity sufficient to excite two higher threshold MUPs (MU3 and MU4). At each increment in the stimulus intensity ( 0 ), 100 consecutive stimuli were delivered of constant current. The sequence was reproducible. Note the increase in the $F$ discharge frequency of MU2 which paralleled the recruitment of MU3 and the subsequent reduction in frequency with recruitment of MU4. Stimulus frequency was one per second.

Interaction between antidromic inputs from single motor fibres to change the frequency of $F$ discharge in motor units was observed in one patient with Guillain-Barré polyneuritis but not in healthy nerves because the thresholds for individual motor fibres overlap too much for the long test stimulus trains required. In Fig. 6 the firing frequencies or indices from $0-100 \%$ are plotted for the first four successive motor units excited by increments in the stimulus intensity. Only the second (MU2) and fourth (MU4) units had F discharges. Recruitment of the third MUP (MU3) increased dramatically the $F$ discharge index of MU2 to almost $50 \%$. Further increments in the stimulus intensity recruited the fourth motor unit, resulting in a significant but reproducible reduction in the firing index of MU2 to below $25 \%$, a frequency that remained remarkably constant despite further increases in the stimulus intensity to excite more motor fibres. Sensory nerve action potentials were absent in this patient. Of course, the possibility could not be excluded that sensory fibre stimulation was responsible for changes in the F firing index of MU2 but this was judged unlikely in view of the lack of evidence for a significant conditioning influence of sensory fibre input on the $F$ discharge of motor units in controls.

RELATION OF MOTOR UNIT POTENTIAL M LATENCY AND SURFACE VOLTAGE TO THE CORRESPONDING F LATENCY

Motor units contributing to the $M$ wave are re-

cruited in an orderly manner from those with lower surface voltage MUPs and longer latency, to those with higher surface voltage MUPs and shorter latencies by increments in the stimulus $\stackrel{?}{\stackrel{?}{\circ}}$ intensity (Kadrie et al., 1976; Kadrie and Brown, 1978). It is not, therefore, unreasonable to expecte that if this relation of direct M MUP latency to surface voltage is maintained over proximal sege ments of the peripheral nerves, and provided the central spinal cord delay is more or less constant for all motor units, this relation would be maintained such that the larger surface voltage $F$ MUPs would have shorter latencies and the converse, lower surface voltage $F$ MUPs longer latencies. Such a relationship was not observed for thenar or hypothenar MUPs (Fig. 7).

Figure 7 illustrates the relationship between the surface voltages and $\mathrm{F}$ latencies for hypothenar MUPs from nine control subjects. There was no significant correlation between the two parameters. For nerve stimulation at the level of the wrist, the latency range of F MUPs was between 1.1 and $7.0 \mathrm{~ms}$ (mean $2.8 \mathrm{~ms}$ ), the corresponding range and mean for F MUPs isolated by stimulation at the level of the elbow being 1.7-2.3 ms (mean $2.0 \mathrm{~ms}$ ).

This evidence suggested a possible breakdown in the relationship between F latency and MUP surface voltage for conduction over proximal segments of the peripheral nerve. This lack of correlation, though not systematically investigated at the time, was apparent in our first investigation of the F discharge (see Fig. 6 of Feasby and Brown 


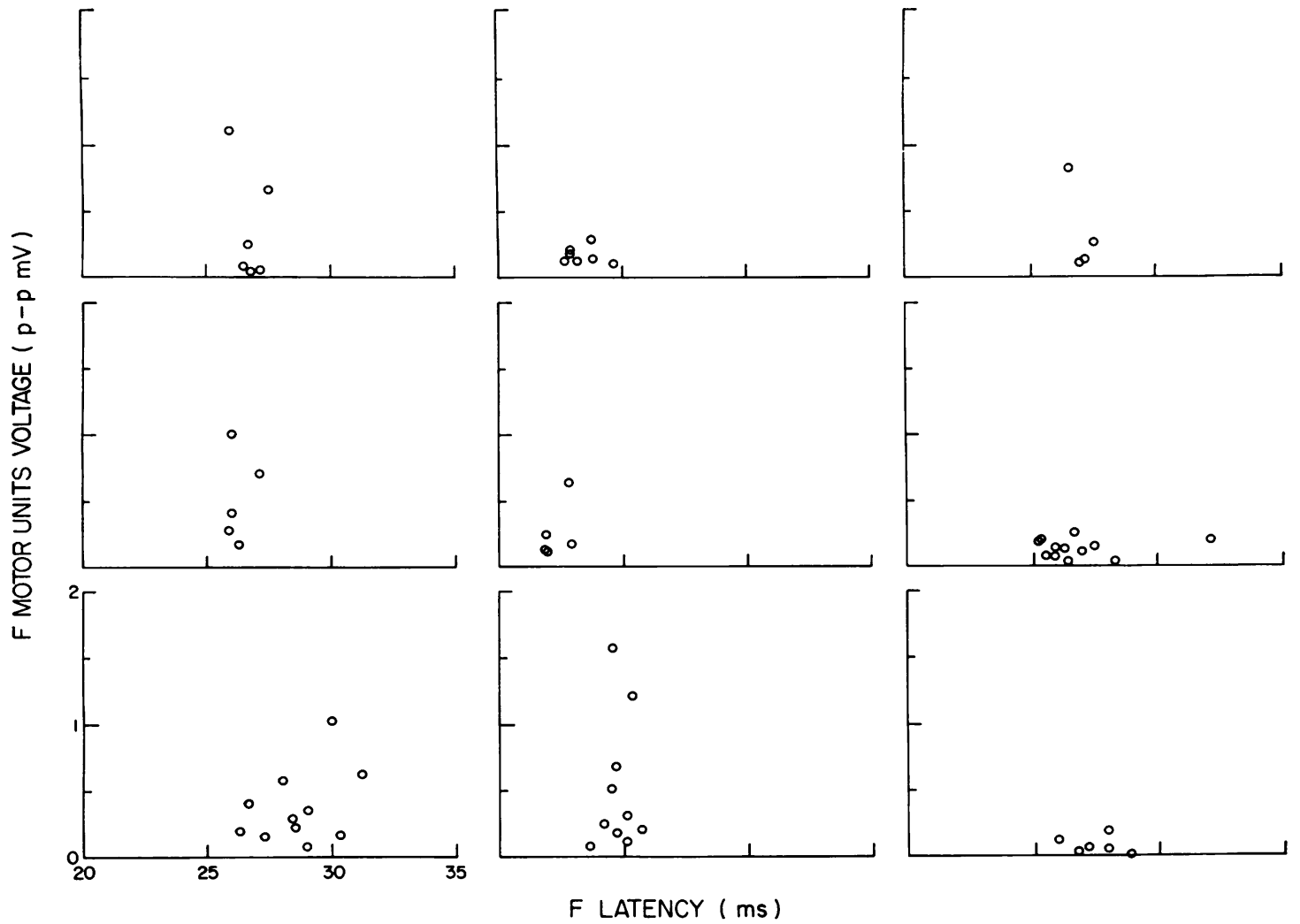

Fig. 7 Hypothenar F MUPs from nine control subjects, obtained by stimulation of the ulnar nerve at wrist level, and over stimulus intensity ranges evoking an M response 10 to $100 \%$ of the maximum compound potential. No significant correlation was observed between MUP F latency and the surface voltage.

Stimulus frequency was one per second.

(1974)). The low $F$ discharge frequency in control MUPs limited to an insignificant number the examples where $\mathbf{M}$ and $\mathbf{F}$ latencies could be compared directly for MUPs recruited at or just above motor threshold. The problem was even more difficult at higher stimulus intensities because the $M$ responses of individual motor units were, of course, lost in the compound $M$ potential. Even the use of the more discriminating single fibre EMG electrode did not make it possible to measure individual MUP M latencies with sufficient precision to permit the comparison of $\mathbf{M}$ and $F$ latencies of motor unit potentials.

\section{Discussion}

The $F$ recurrent discharge is an unnatural event that results from the reactivation of the motoneurone initial segment or first node consequent to antidromic invasion of the soma-dendritic tree by an antidromic pulse originating at a more peripheral location on the motor axon (Eccles, 1955; Dawson and Merton, 1956; Thorne, 1965; Gassel and Wiesendanger, 1965; McLeod and Wray, 1966; Bergmans, 1970; Trontelj, 1973a). The variability observed in the latency and pattern of the F discharge results from the fact that it represents the sum of the relatively infrequent $F$ discharges in a limited fraction of the total population of motor units, the latency for any particular MUP F discharge being quite fixed (Bergmans, 1970; Trontelj, 1973a; Trontelj and Trontelj, 1973; Feasby and Brown, 1974; Yates and Brown, 1977; Schiller and Stalberg, 1978).

Measurement of the $F$ discharge latencies has been suggested as a practical method for measuring motor conduction times over proximal segments of human peripheral nerves (Kimura, 1974; Kimura et al., 1974; Kimura and Butzer, 1975; King and Ashby, 1976; Eisen et al., 1977a,b). Criti- 
cal acceptance, however, of measurements of proximal conduction times using this method make it necessary to be certain that the MUPs used to measure the $F$ latency are the shortest conduction time MUPs and more evidence that the central delay for a motor unit $\mathrm{F}$ discharge is close to 1.0 ms for all units.

It was evident in this and previous investigations that, in healthy humans, the expectation for MUP $F$ discharge is one in 10 or less, at least at motor threshold (Bergmans, 1970; Feasby and Brown, 1974; Yates and Brown, 1977; Schiller and Stalberg, 1978) only about one in four motor units having an $F$ discharge in response to 100 consecutive stimuli delivered at one per second. The fraction of motor units with an $\mathrm{F}$ discharge varied from zero to close to $50 \%$ of motor units, depending on the subject and the length of the stimulus trains.

The proportion of motor units with $\mathrm{F}$ discharges as expected increased the longer the stimulus train (Bergmans, 1970; Schiller and Stalberg, 1978). Obviously, in the clinical situation, there is a practical limit to the length of the test train. If the test train is too short, for example 10 stimuli or less (Eisen et al., 1977a,b), the number of MUPs included in the $\mathrm{F}$ discharge could be too limited to be representative in surface voltage or latency of the motor units with the shortest conduction time. On the other hand, if the stimulus trains are too long, the test may be too uncomfortable and require too much time to be practical. In pathological nerves occasional motor units have much higher $\mathrm{F}$ discharge frequencies (Brown and Yates, unpublished). From our observations, a practical limit to the stimulus train was 50 stimuli. A longer train did not significantly increase the total number of units with $\mathrm{F}$ discharges; a shorter train included too few MUPs in the F discharge to be representative.

There was no evidence in this investigation that the probability of $F$ discharge was related to the MUP surface voltage or latency. This point is important because MUPs with higher expectations of $F$ discharge have the best chance of being recorded using short stimulus trains (10 or less) commonly used in clinical test situations. The level of the stimulation in the upper limb, wrist, or elbow made no difference to the percentage of motor units with $F$ discharges.

The questions about the recruitment order of motor units into the direct $M$ and late $F$ discharges were intriguing. It was previously reported that motor units with larger MUPs were recruited into the $F$ discharge at higher stimulus intensities (Feasby and Brown, 1974) and that in the direct
M discharge MUPs were recruited from small to larger surface voltage and longer to shorter latency by increments in the stimulus intensities delivered to the nerve (Kadrie et al., 1976; Kadrie and Brown, 1978). In this investigation, more evidence that the larger MUPs are recruited into the $F$ discharge at higher stimulus intensities was presented. The increase in the mean MUP surface voltage evoked by successively higher stimulus intensity levels was, in part, the result of recruitment of units with larger MUPs but reflected, too, the dropout of smaller MUPs recruited at lower stimulus intensities. It follows from these observations that supramaximal stimulation probably biases the F MUP population to larger MUPs, a happy bias if, in fact, these MUPs had turned out to have the shortest latencies.

What mechanisms explain the effect of the stimulus intensity on the $\mathrm{F}$ discharge? In view of the comparative excitabilities of the larger sensory fibres and motor fibres evident in Fig. 4, and the not unreasonable view that inputs from higher threshold sensory fibres could not reach the ventral horn in time to change motoneurone excitability before the arrival of the antidromic motor inputs, there must be mechanisms whereby antidromic motor inputs in motor fibres can changes the probability of unit F discharge. Such an inter- $\varrho$ action was not observed for single MUPs with Fo discharges at motor threshold in the few examples in which it has been tested in this investigation and from the previous experience of Bergmans (1970).

In disease states, however, as in the example of Guillain-Barré polyneuritis illustrated by Fig. 6 , evidence was presented to support the view that recruitment of individual MUPs could change the probability of $F$ discharge in a single MUP, being capable both of increasing and decreasing that expectation. Obviously, if the recruitment of single MUPs is to change the frequency of MUP F discharge, the inputs from conditioning MUPs must reach the motoneurone in time to change the neurone excitability at or just before invasion by its own antidromic action potential. Mechanisms for changing the excitability of other motoneurones by antidromic inputs include recurrent inhibition by Renshaw cells, and recurrent direct excitation (transynaptic) (Cullheim et al., 1977; Gogan et al., 1977) interaction between motoneurones (Ryall et al., 1972) membrane potential changes induced by changes in the extracellular potentials from the ventral horn being a less likely source (Eccles et al., 1954; Eccles, 1955).

In the Methods section, the third criterion necessary for accepting unit discharges as true 
single MUPs was based on the assumption that synchronous or near-synchronous coupling of $F$ discharges in two or more motor units was unlikely, the probabilities for $F$ discharge in units being independent. Clearly this was not always the case. Changes in the stimulus intensity were shown to increase or reduce the $F$ discharge frequency in some MUPs. Moreover, in the one pathological case cited, there was a change in $F$ discharge frequency brought about by the recruitment of two other MUPs. It turned out, however, that despite looking for such a striking interaction in other MUPs, this was the only example observed. No such interaction was observed for motor units excited at or just above motor threshold. In addition, even the changes in $\mathrm{F}$ discharge frequency in control nerves at higher stimulus intensities were not of an order to invalidate the third criterion.

Sensory input, as mentioned, was an unlikely candidate to change the motor unit $F$ frequency because most of the changes in frequency are observed at stimulus intensities supramaximal for the antidromic digital sensory nerve action potential and digital sensory fibre inputs timed to precede the arrival of the antidromic motor input to the ventral horn failed to change the frequency of F MUP discharge in the MUPs tested.

Unexpected was the absence of a significant relationship between MUP surface voltage and F latency, there being such a relationship between surface voltage and latency for MUPs in the direct $M$ response (Kadrie et al., 1976; Kadrie and Brown, 1978). This lack of a relationship between F MUP latency and MUP surface voltage was hard to explain. One possible explanation was that the latency of MUPs in the F discharge could not be measured properly, particularly at high stimulus intensities where $F$ discharges were superimposed on large positive deflections from the direct $M$ response. The latter probably limited the accuracy of the latency measurements but the resultant errors would be true for MUPs of all sizes and, in our view, were unlikely to account for the lack of correspondence between MUP surface voltage and $F$ latency observed in this investigation.

If technical factors could not fully account for the breakdown in the relationship between MUP F latency and surface voltage, the breakdown must occur at the level of the proximal peripheral nerves, roots, or spinal cord. The conduction velocities in the brachial plexus and nerve roots have been reported to be less than across more distal peripheral nerve segments in the baboon in situations where the lengths of the various seg- ments could be measured accurately and the temperature controlled (Clough et al., 1968).

Another possibility was that there could have been differences between motor units in the central delay for motoneurone reactivation sufficient to account for the lack of a relation between MUP surface voltage and $F$ latency. There has been no systematic investigation of the $F$ discharge in mammalian motoneurones, and certainly no systematic investigation of possible differences in the central latency from one motor unit to another, and no attempt to relate the frequency of $F$ discharge or the proximal conduction velocity to the motor unit or its functional type or MUP size. Eccles (1955) has stated that the central delay must be close to $1.0 \mathrm{~ms}$ but there are no records illustrating this directly (Eccles, 1971 personal communication). In invertebrate giant neurones, central delays much longer than $1.0 \mathrm{~ms}$ have been measured (Tauc, 1962a,b).

Therefore, there are probably unknown factors operating at the level of the proximal peripheral nerve roots or spinal cord that bring into question the validity of the $\mathrm{F}$ discharge method as an accurate measure of proximal peripheral nerve conduction time. To use the $F$ response to measure proximal conduction times requires that all motor units have a more or less uniformly short central delay in health and disease and the certainty that the shortest latency $F$ motor units recruited using supramaximal nerve stimulation truly represent the maximum conduction velocities for units over that segment. In view of the fact, particularly for short stimulus trains, that only a very limited proportion of motor units contribute to the $F$ discharge, it seems reasonable to question the validity of such latency measurements as indices of proximal conduction time until further experimental evidence is forthcoming.

We would like to express our appreciation for the patience and diligence of Mrs T. Stewart and Mrs N. McGregor in the preparation of this manuscript, and to Mr George Moogk for the art work.

\section{References}

Bergmans, J. (1970). The Physiology of Single Human Nerve Fibres. Vander: Brussels.

Brown, W. F., and Milner-Brown, H. S. (1976). Some electrical properties of motor units and their effects on the methods of estimating motor unit numbers. Journal of Neurology, Neurosurgery, and Psychiatry, 39, 249-257.

Clough, J. F. M., Kernell, D., and Phillips, C. G. (1968). Conduction velocity in proximal and distal 
portions of forelimb axons in the baboon. Journal of Physiology, 198, 167-178.

Cullheim, S., Kellerth, J. O., and Conradi, S. (1977). Evidence for direct synaptic interconnections between cat spinal $\alpha$-motoneurones via the recurrent axon collaterals: a morphological study using intracellular injection of horseradish peroxidase. Brain Research, 132, 1-10.

Dawson, G. D., and Merton, P. A. (1956). "Recurrent" discharges from motor neurones. Proceedings of the 20th International Congress of Physiology. Bruxelles.

Eccles, J. C. (1955). The central action of antidromic impulses in motor nerve fibres. Pflügers Archiv für die gesamte Physiologie des Menschen und der Tiere, 260, 385-415.

Eccles, J. C., Fatt, P., and Koketsu, K. (1954). Cholinergic and inhibitory synapses in a pathway from motor-axon collaterals to motoneurones. Journal of Physiology, 126, 524-562.

Eisen, A., Schomer, D., and Melmed, C. (1977a). An electrophysiological method for examining lumbosacral root compression. Canadian Journal of Neurological Sciences, 4, 117-123.

Eisen, A., Schomer, D., and Melmed, C. (1977b). The application of F-wave measurements in the differentiation of proximal and distal upper limb entrapments. Neurology (Minneapolis), 27, 662-668.

Feasby, T. E., and Brown, W. F. (1974). Variation of motor unit size in the human extensor digitorum brevis and thenar muscles. Journal of Neurology, Neurosurgery, and Psychiatry, 37, 916-926.

Gassel, M. M., and Wiesendanger, M. (1965). Recurrent and reflex discharges in plantar muscles of the cat. Acta Physiologica Scandinavica, 65, 138142.

Gogan, P., Gueritaud, J. P., Horcholle-Bossavit, G., and Tyc-Dumont, S. (1977). Direct excitatory interactions between spinal motoneurones of the cat. Journal of Physiology, 272, 755-767.

Kadrie, H. A., and Brown, W. F. (1978). Neuromuscular transmission in single human motor units. Journal of Neurology, Neurosurgery, and Psychiatry, 41, 193-204.

Kadrie, H. A., Yates, S. K., Milner-Brown, H. S., and Brown, W. F. (1976). Multiple point stimulation of ulnar and median nerves. Journal of Neurology, Neurosurgery, and Psychiatry, 39, 973-985.

Kimura, J. (1974). F wave velocity in the central segment of the median and ulnar nerves. Neurology (Minneapolis), 24, 539-546.

Kimura, J., and Butzer, J. F. (1975). F wave conduction velocity in Guillain-Barré syndrome. Archives of Neurology (Chicago), 32, 524-529.
Kimura, J., Butzer, J. F., and Van Allen, M. W. (1974). F wave conduction velocity between axilla and spinal cord in the Guillain-Barré syndrome. Transactions of the American Neurological Association, Boston, 99, 52-62.

King, D., and Ashby, P. (1976). Conduction velocity in the proximal segments of a motor nerve in the Guillain-Barré syndrome. Journal of Neurology, Neurosurgery, and Psychiatry, 39, 538-544.

McLeod, J. G., and Wray, S. H. (1966). An experimental study of the $F$ wave in the baboon. Journal of Neurology, Neurosurgery, and Psychiatry, 29, 196-200.

Ryall, R. W., Piercey, M. F., Polosa, C., and Goldfarb, J. (1972). Excitation of Renshaw cells in relation to orthodromic and antidromic excitation of motoneurones. Journal of Neurophysiology, 35, 137-148.

Schiller, H. H., and Stalberg, E. (1977). The F wave studied by single fibre electrcmyography. Electroencephalography and Clinical Neurophysiology, 42, 130.

Schiller, H. H., and Stalberg, E. (1978). F responses studied with single fibre EMG in normal subjects and spastic patients. Journal of Neurology, Neurosurgery, and Psychiatry, 41, 45-53.

Tauc, L. (1962a). Site of origin and propagation of spike in the giant neuron of Aplysia. Journal of General Physiology, 45, 1077-1097.

Tauc, L. (1962b). Identification of active membrane areas in the giant neuron of Aplysia. Journal of General Physiology, 45, 1099-1115.

Thorne, J. V. (1965). Central responses to electrical activation of the peripheral nerves supplying the intrinsic hand muscles. Journal of Neurology, Neurosurgery, and Psychiatry, 28, 482-495.

Trontelj, J. V. (1973a). A study of the F responses by single fibre electromyography. In New Developments in Electromyography and Clinical Neurophysiology, vol. 3, pp. 318-322. Edited by J. E. Desmedt. Karger: Basel.

Trontelj, J. V. (1973b). A study of the H reflex by single fibre EMG. Journal of Neurology, Neurosurgery, and Psychiatry, 36, 951-959.

Trontelj, J. V., and Trontelj, M. (1973). F responses of human facial muscles. A single motor neurone study. Journal of the Neurological Sciences, 20, 211-222.

Yates, S. K., and Brown, W. F. (1977). The F discharge in normal and abnormal nerves. Proceedings of the 11th World Congress of Neurology, Amsterdam. 